\section{Virtual reality simulator for vitreoretinal surgery}

D Verma', D Wills² and M Verma²

\begin{abstract}
Aim To develop computer simulation of steps in vitreoretinal surgery using virtual reality technology.

Material and methods A workstation with three-dimensional position tracking stylus was attached to a Pentium II desktop PC with a graphic accelerator. Computer algorithms were developed using Open GL and Microsoft Visual $\mathrm{C}++$ languages to control the interaction and update the visual feedback tracking the instruments. Soft tissue computer modelling was carried out to mimic the removal of a vitreous opacity. Lens touch with the instruments was also detected.

Mathematical modelling to allow for lens distortion was taken into account.

Results A virtual reality computer model has been developed that can simulate initial steps of vitreoretinal surgery. Soft tissue modelling of the vitreous opacity and its removal by the vitrector was successfully simulated. The movements of the active and passive instruments in the dummy eye corresponded to the movements on the computer screen. On evaluation of the system, there was a minimal but discernable time lag between the stylus movement and the visual feedback. There was no tactile feedback when the lens touch was simulated. No further complex vitreoretinal surgery simulation was possible at this stage. Eye (2003) 17, 71-73. doi:10.1038/

sj.eye. 6700166
\end{abstract}

Keywords: virtual reality; vitreoretinal surgery; simulated surgery; vitreoretinal simulator

\section{Background}

Computer-driven flight simulators have been in existence for many years, contributing a great deal to the training and recertification of pilots without direct risk to life or limb. ${ }^{1}$ Surgical skills have traditionally been learnt on live patients under supervision, but increasing pressures on maintaining consistent quality and reducing possible risks due to the 'learning curve' have made this method unacceptable in early stages of surgical training. Simulators in clinical surgery were subsequently developed for the same reason. ${ }^{2}$ With further advances in computer technology and increasing complexity of key hole surgery, virtual reality simulators seem to be a better alternative, attracting further research in this field. ${ }^{3-5}$ Computer simulators for training in otolaryngology ${ }^{6}$ and retinal laser therapy ${ }^{7}$ have been found to be superior to conventional training. Computer-simulated eye surgery for the anterior segment has been developed as a novel teaching method for residents and practitioners without any risk to the patients. ${ }^{8}$ Vitreoretinal surgery is a complex surgery requiring a high level of dexterity on the part of the surgeon and any error during the learning curve can result in irreversible damage to the sensitive structures of the eye. This study was designed to assess the feasibility of a similar approach for vitreoretinal surgery.

\section{Materials and methods}

Various components for the virtual reality simulator system included:

1. The operating work station comprising of dummy eye ball (a ping-pong ball) with hand-held three-dimensional position tracking stylus incorporated in the thin rigid bars used as active and passive instruments (Figure 1).

2. Computer models of anatomy and of parts of surgical instruments visible inside the eye using desktop PC Pentium II with graphic accelerator (Figures 2a-d).

3. Magnetic position Polhemus tracking system (Figure 3 ).

Computer algorithms were developed using Open GL and Microsoft Visual C ++ languages to control the interaction and update the visual feedback tracking the instruments. The $x, y, z$ coordinates on the screen were related
${ }^{1}$ Department of Ophthalmology Hull Royal Infirmary Hull, UK

${ }^{2}$ Department of Computer Science

University of Hull

Hull, UK

Correspondence:

D Verma

Doheny Retina Institute 1450 San Pablo Street DEI - 3611 Los Angeles CA 90033-3699 USA

Tel: + 13234426571

Fax: + 13234426460

E-mail: dverma@

dei.hsc.usc.edu

Received: 30 July 2001 Accepted: 8 March 2002 


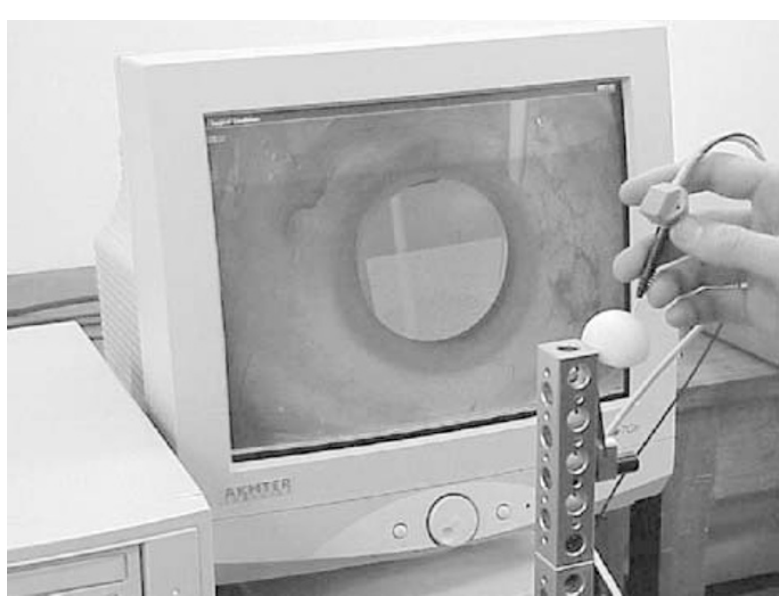

Figure 1 Basic simulator set-up.

to the $x, y, z$ of the hand movements by visual alignment using the magnetic position Polehemus tracking system. Soft tissue computer modelling was used to depict a vitreous opacity in the eye (Figure 2a). Tracking and programming allowed simulated entry of the cutter and its interaction with the vitreous opacity. Figure $2 b$ demonstrates piecemeal removal of the opacity with the cutter under direct microscope illumination. Light pipe entry was simulated and tracked via another entry site

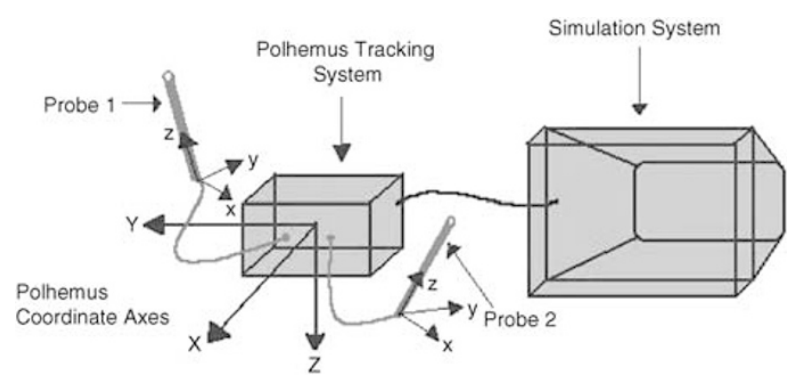

Figure 3 Magnetic position of the Polhemus tracking system.

(Figure 2c) and further removal of the opacity could then be carried out simulating endo-illumination during vitreoretinal surgery (Figure $2 \mathrm{~d}$ ). Lens touch with either instrument was detected by the system and depicted visually as the computer screen turning red, giving instant feedback to the surgeon. Mathematical modelling to allow for lens distortion was taken into account (Figure 4).

\section{Results}

A virtual reality computer model has been developed that can simulate the initial steps of vitreoretinal surgery.

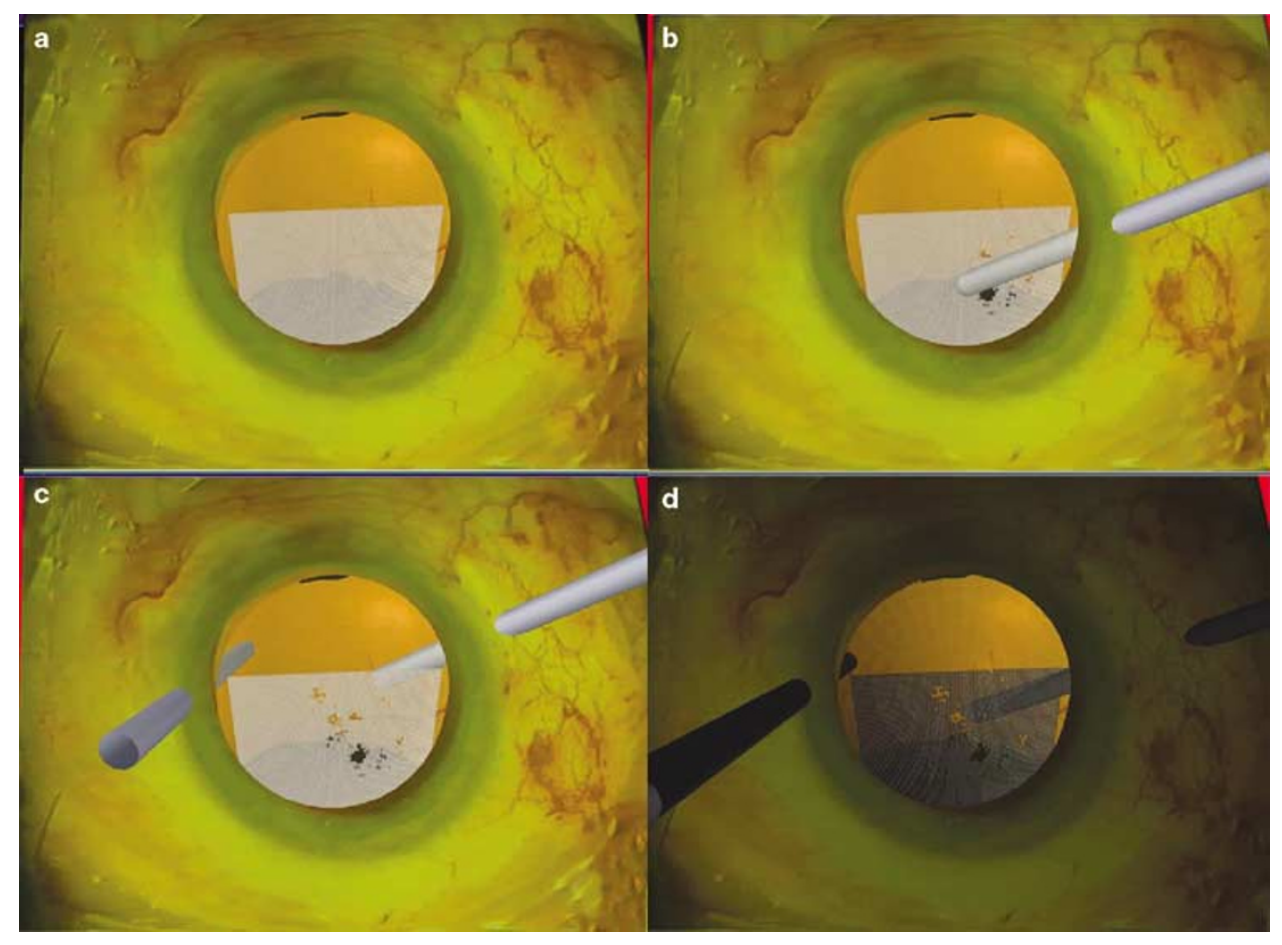

Figure 2 Simulation of steps of vitreous surgery: (a) Simulation of vitreous opacity; (b) Insertion of active instrument with simulation of piecemeal removal of the opacity under direct illumination; (c) Insertion of passive instrument; (d) Removal of vitreous opacity with endo-illumination. 


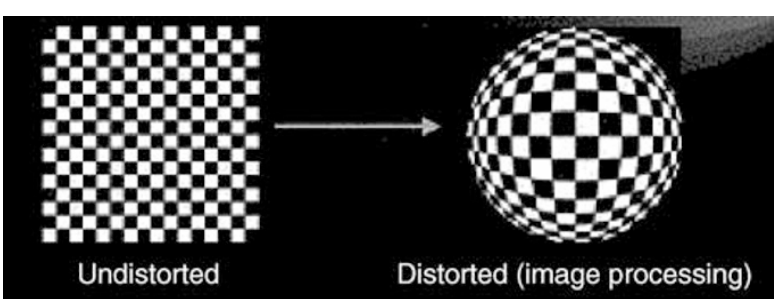

Figure 4 Mathematical model of lens distortion.

Soft tissue modelling of the vitreous opacity resulted in simulation of its removal under direct and retroillumination. The movements of the active and passive instruments in the dummy eye corresponded to the movements on the computer screen, although the entry sites visible on the screen were closer than those in the model eye. On evaluation of the system, there was a minimal but discernable time lag between the stylus movement and the visual feedback. Even though time lag was in microseconds, it was disconcerting to the evaluating surgeon. There was no tactile feedback when the lens touch was detected by a visual signal. There was no stereoscopic viewing of the simulated surgery and no further complex vitreoretinal surgery simulation was possible at this stage.

\section{Discussion}

A carpenter learns his skills on wood that is never displayed. Pilots have been learning and improving their skills on computer-assisted simulators for many years. There is increasing demand for surgical simulators that can be used for the early stages of surgical training without risk to the patients. There is also a possibility of carrying out objective assessment of surgical dexterity using such simulators. ${ }^{9}$ Rapid advances in computer technology and virtual reality make it possible to develop these systems on relatively inexpensive hardware. ${ }^{10}$ This study has shown that it is technically feasible to simulate vitreoretinal surgery in a virtual environment, although it would require further refinements in technology for it to become a viable alternative for training surgeons in the early stages. Tactile feedback needs to be incorporated for the system to be effective as a training tool. It has been shown that surgeons' tool force/torque signature is very important in the evaluation of minimally invasive surgery. ${ }^{11}$ We are now planning to use Phantom ${ }^{\mathrm{TM} 12}$ technology to allow full force feedback data. The system also needs to be fully assessed in comparison to other available 'wet-lab'-type systems as the validity of virtual reality surgical simulators has recently been questioned. ${ }^{13}$ The advantages of virtual reality systems over currently available wet-lab-type eye models are the ability to create any number of surgical scenarios within the same system by computer programming, as well as the possibility of accurate numerical assessment of the skills by the feedback mechanism. Hikichi et al have recently reported a similar simulator for vitreous surgery but technical details are lacking in the paper. ${ }^{14}$ We are also aware of a commercial development of a virtual reality simulator by a German company, but this has not been the subject of a research paper. Collaborative efforts are now under way between our groups to develop the system further so as to provide a 'near live' experience of vitreoretinal surgery to the trainees. The problem of tactile feedback is currently being solved by a collaborative project with the Department of Engineering at The Johns Hopkins University using advanced computer technology and is the subject of a separate paper. With recent developments in relation to clinical governance and revalidation, virtual reality simulation may become a useful tool for training and assessment in the near future.

\section{References}

1 Haber RN. Flight simulation. Sci Am 1986; 255: 96-103.

2 Hoffman H, Vu D. Simulators in clinical surgery. J Audiovis Media Med 1997; 20(4): 178-179.

3 Satava RM. Virtual reality surgical simulator. The first steps Surg Endosc 1993; 7(3): 203-205.

4 Pai G. Virtual reality: teaching tool of the twenty-first century? Acad Med 1997; 72(12):1076-1081.

5 Lange T, Indelicato DJ, Rosen JM. Virtual reality in surgical training. Surg Oncol Clin N Am 2000; 9(1): 61-79; vii. Review.

6 Kuppersmith RB, Johnston R, Jones SB, Jenkins HA, Bobby R. Alford. Virtual reality surgical simulation and otolaryngology. Arch Otolaryngol Head Neck Surg 1996; 122(12): 1297-1298.

7 Peugnet F, Dubois P, Rouland JF. Virtual reality versus conventional training in retinal photocoagulation: a first clinical assessment. Comput Aided Surg 1998; 3(1): 20-26.

8 Sinclair MJ, Peifer JW, Haleblian R et al. Computersimulated eye surgery: a novel teaching method for residents and practitioners. Ophthalmology 1995; 102: 517-521.

9 Smith SG, Torkington J, Darzi A. Objective assessment of surgical dexterity using simulators. Hosp Med 1999; 60(9): 672-675.

10 Tseng CS, Lee YY, Chan YP, Wu SS, Chiu AW. A PC-based surgical simulator for laparoscopic surgery. Stud Health Technol Inform 1998; 50: 155-160.

11 Rosen J, MacFarlane M, Richards C, Hannaford B, Sinanan M. Surgeon-tool force/torque signatures - evaluation of surgical skills in minimally invasive surgery. Stud Health Technol Inform 1999; 62: 290-296.

12 http://www.com/WEB/PRODUCTS/PHANTOM/HTM.

13 Reznick RK.Virtual reality surgical simulators: feasible but valid? J Am Coll Surg 1999; 189(1): 127-128.

14 Hikichi T, Yoshida A, Igarashi Syo, Mukai N, Harada M, Muroi K et al. Vitreous surgery simulator. Arch Ophthalmol 2000; 118: 1679-1681. 